\title{
Erratum: Three-body hypernuclei in pionless effective field theory [Phys. Rev. C 100, 034002 (2019)]
}

\author{
F. Hildenbrand and H.-W. Hammer $\odot$
}

(Received 24 July 2020; published 10 September 2020)

DOI: 10.1103/PhysRevC.102.039901

In the calculation of the $\Lambda d$ scattering amplitude for physical $M_{\Lambda}$, the normalization factor for $M_{\Lambda}=M$ was used [cf. Eq. (10)]. Correcting this error, $k \cot \delta$ changes by a factor of $(3-y) /[3(1+y)] \approx 0.89$. Consequently, the value for $a_{\Lambda d}^{y=0.086}$ in Eq. (29) changes to $a_{\Lambda d}^{y=0.086}=15.4_{-2.3}^{+4.3} \mathrm{fm}$. The wrong normalization factor also impacts Figs. 5 and 6 , but the qualitative behavior is unchanged. Corrected versions of Figs. 5 and 6 are given below. All other results and equations remain unchanged.

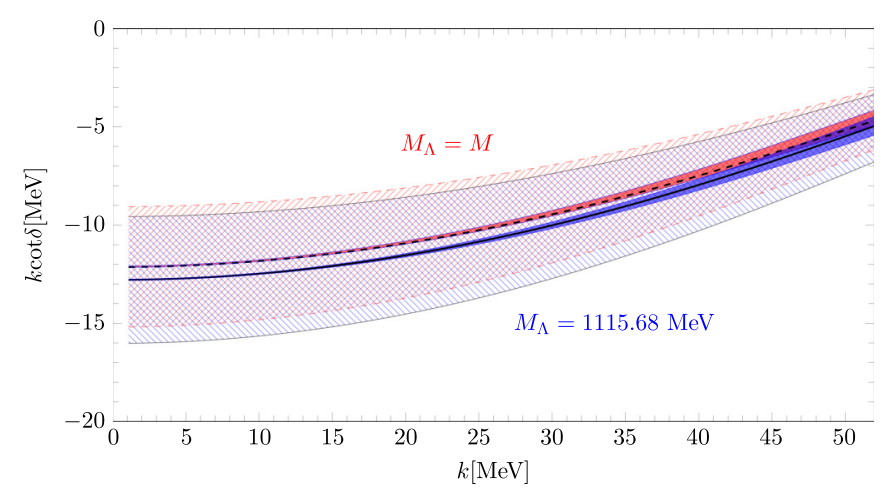

FIG. 5. $\Lambda-d$ scattering phase shifts for $y=0$ (dashed black line) and physical value of the $\Lambda$ mass (solid black line). The dark blue and red bands represent the sensitivity to a variation of the chiral effective field-theory (EFT) input scattering lengths by $15 \%$, whereas the blue and red hatched bands give an estimate of the EFT error.

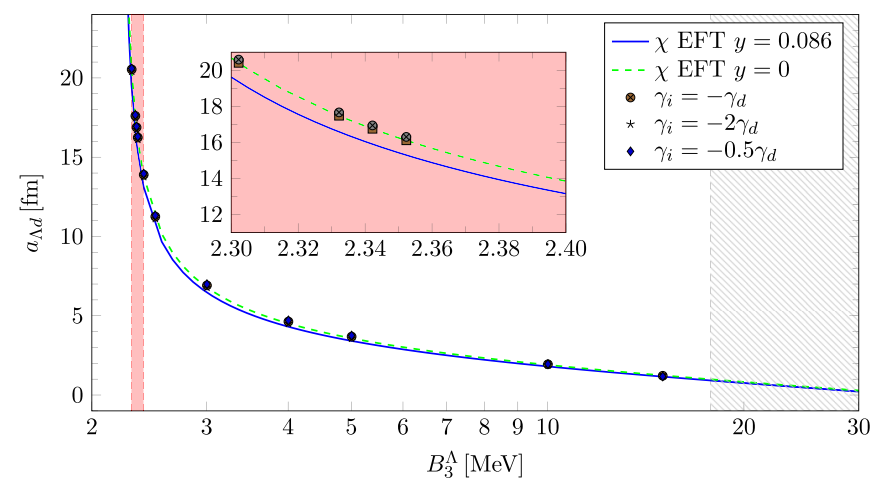

FIG. 6. Phillips line for the hypertriton for $y=0$ (dashed green line) and physical $\Lambda$ mass (solid blue line). In the gray shaded area, the EFT description breaks down, whereas the red shaded area represents the physical binding energy region and is enhanced in the inset. The different black symbols illustrate the sensitivity to changes in $\gamma_{i}=1 / a_{i}$, where $i=1,3$. 